\title{
Public Health - Öffentliche Gesundheit als interdisziplinäre Herausforderung der Zukunft
}

\author{
Erster Universitätslehrgang, der Public Health, körperliche Aktivität, \\ Sport und Ernährung integriert
}

Wenn man in Internet-Suchmaschinen den Begriff „Public Health“ eingibt, so finden sich bei weltweiter Abfrage zirka 220 Millionen Einträge. Beim Suchbegriff "Beatles" erscheinen „nur“ knapp 55 Millionen Einträge. Welche Bedeutung ist also einem Begriff beizumessen, der viermal so oft wie die Beatles Erwähnung findet und ins Deutsche als „Öffentliche Gesundheit" übersetzt wird?

Die Geschichte von Public Health ist eng verwoben mit der Geschichte der Medizin, des sozialen Sicherungssystems, der Gesundheitsverwaltung und der Sozial- und Politikgeschichte im Allgemeinen. Im Gesundheitsbereich hat sich Public Health zu einer Managementdisziplin zur Leitung und Steuerung der Krankenversorgung und Gesundheitsvorsorge entwickelt. Eine multidisziplinäre Bündelung und Vernetzung ermöglicht die Erfassung gesundheitsrelevanter Daten, die Aufdeckung und Analyse von Einflüssen von Gesellschaft und Umwelt auf Gesundheit und Krankheit, sowie die Entwicklung von kosteneffizienten, bevölkerungsbezogenen Maßnahmen für die Prävention, Gesundheitsförderung, Verbesserung der medizinischen Versorgung, Änderung des Verhaltens oder Lebensstils und Kontrolle der Umweltbedingungen.

Das Ziel von Public Health ist demnach auch, dementsprechende Initiativen in Forschung, Bildung und Öffentlichkeitsarbeit zu verbessern. Im 20. Jahrhundert wurden diesbezüglich insbesondere im angloamerikanischen Raum erste universitäre Forschungsund Ausbildungsprogramme entwickelt, wie beispielsweise die School of Public Health in Harvard im Jahre 1922.

In Österreich sind der Begriff „Public Health“ und seine umfassende Bedeutung vergleichsweise wenig bekannt und seine Lehre und Forschung an den medizinischen Universitäten nur in Teilbereichen institutionalisiert.
Eine beachtliche Vorreiterrolle spielt der Universitätslehrgang Master of Public Health Vienna, der unter der Schirmherrschaft der Medizinischen Universität Wien in Kooperation mit der Universität Wien im Jahr 2005 implementiert wurde.

Unter der wissenschaftlichen Leitung der Sozialmedizinerin Univ. Prof. Dr. Anita Rieder seitens der Medizinischen Universität Wien und dem Sportmediziner Univ. Prof. Dr. Norbert Bachl seitens der Universität Wien wurde gemeinsam mit der organisatorischen Lehrgangsleitung, dem Sport- und Präventivmediziner Dr. Piero Lercher und dem Sportwissenschaftler Mag. HansChristian Miko, ein Curriculum zusammengestellt, das eine fundierte Qualifikation mit hoher Praxisorientierung darstellt.

Der Schwerpunkt der berufsbegleitenden Ausbildung liegt neben den klassischen Public-Health-Grundlagen, im Bereich der Lebensstilmedizin und Prävention- und Gesundheitsförderung mit besonderer Berücksichtigung der Leistungs-, Ernährungs- und Sportmedizin in Hinsicht auf Kompetenzen zur nachhaltigen Umsetzung des erworbenen Wissens in der Praxis und schließt damit eine wichtige Bedarfslücke - national aber auch international.

Diese Kombination von klassischen Public-Health-Inhalten mit sportund ernährungsmedizinischen Fachwissen macht bereits international Schule. Seit der Implementierung des Lehrganges im Jahr 2005 nahmen Teilnehmer/innen aus 10 Nationen teil (u.a. Australien, China, Arabische Emirate, Kolumbien, USA und einige Nachbarländer aus Österreich). Einige Absolventen/innen machten bereits eindrucksvolle Karrieren, wurden beispielsweise in das Beratungsteamdes Gesundheitsministers aufgenommen oder erhielten, teilweise noch vor Abschluss des Lehrgangs, Jobangebote bei renommierten Instituten oder Organisationen.

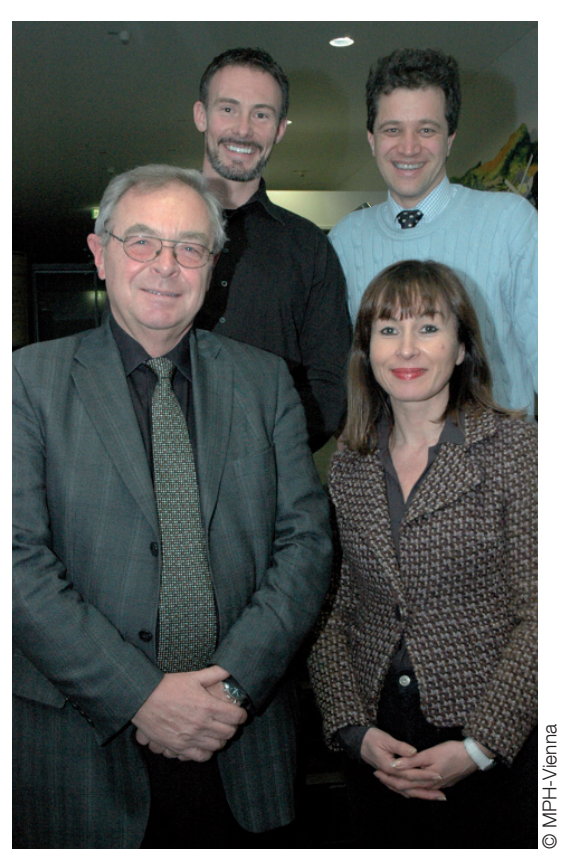

Das Leitungsgremium:

Univ.-Prof. Dr. Anita Rieder (v.r.), Univ.-Prof. Dr. Norbert Bachl (v.l.), Dr. Piero Lercher (h.r.) und Mag. Hans-Christian Miko (h.I.)

In Anlehnung an die große Tradition und internationale Geltung der Wiener Medizinischen Schule entwickelt sich zwischen den nationalen und internationalen Referenten/innen und den Lehrgangsteilnehmer/innen und Absolventen/innen ein wichtiges Netzwerk zur Bewältigung vieler Aufgaben im Gesundheitsbereich und zur Lösung von Krisen im Gesundheitssystem. 